\title{
Optimizing Temporal Business Opportunities
}

\author{
Rola Y. M. Mohammed ${ }^{1}$ \\ ${ }^{1}$ Alliance Business School, University of Manchester, Manchester, UK \\ Correspondence: Rola Y M Mohammed, Alliance Business School, University of Manchester, Manchester, UK. \\ E-mail: rymasud@outlook.com
}

Received: September 2, 2020

Accepted: September 28, 2020

Online Published: October 21, 2020

doi:10.5539/ijbm.v15n11p104

URL: https://doi.org/10.5539/ijbm.v15n11p104

\begin{abstract}
The objective of this paper is to detail preliminary work revolving around modeling. It provides understanding and underpinning implementation procedures of dynamics of large-scale events with Hajj examples, where a large population of people is contained for a significantly long but limited period within certain areas. It is essential to note further that the motivation behind this subject's discussion could also be fueled by sales, inquiries, or security concerns. However, knowledge emergence on service point procedures implementation suggests that service points implementing data are extinct, and this is obliged to implement the next feature. As such, there is a critical need to reform a process and how to analyze the work.

Developing this literature report requires extensive use of factual data for accuracy; as such, data mining and simulation techniques will be essential in explaining what services are needed. The simulation techniques used herein incorporate several databases targeting to exploit the advantage of proficiency in predicting distribution demand for population points based on available current estimates. Henceforth, data mining, in this case, is used to inform intelligent decision making on investing in services points as pushed for by customers' demand.
\end{abstract}

Keywords: large-scale event, temporal service point, system dynamics, simulation, geographic information system (GIS), data mining algorism, decision making

\section{Introduction}

It is not debatable on the fact that the Olympic Games are among the most complex events that require operational organization based on their large-scale size. These games' general format is strict adherence to a four-year cycle and takes place in a specific host country. Often, the games last for 16 days, and attendance from local communities and abroad could be as high as 5 million, and some of the attendance could stay in the arena for as much as the entire time hence the need for prowess and efficiency in hosting the event (Loucopoulos et al., 2003). On the other hand, in Saudi Arabia, Hajj is a similarly large scale event and takes place in Makkah. This event is annual and attracts a Muslim faith population worldwide and stays in the specified location for up to five days (Brdesee, 2013). The specified host locations with predetermined host dates and time and the complexity of the event accounting on the attendance number further suggest that professional event preparation is worth discussing it here.

Henceforth, the Olympic Games and Hajj share a common similarity of hosting a significantly large population for a commendable time in a fixed location and area size. Such populations in these events require the efficient provision of critical resources such as sustainable food and information, high-security beverages, among other goods, a phenomenon that can result in a crisis in logistics. This aspect tables the demand for raising selling points for food products and checkpoints for security services provision (Beis et al., 2006). Notably, these services' nature has varying requirements depending on the nature of the intended service provision. It is evident that a food serving point would require chefs and kitchen accessories, while a security inspection point would require inspection sensors and security guards, among other security-related items (Almunawar, Susanto, \& Anshari, 2013). Variability of the resources required in such large events provides expansive opportunities, and their in-depth understanding is critical for selecting a location that will facilitate their optimal exploitation and in a manner that will provide optimum consumer or customer satisfaction.

The selection of a location that would facilitate optimum exploitation of such large-scale events is linked to several inherent challenges due to the event's temporal nature, the flow of people, and other economic variables worth considering on investments (Marketing, \& Arabia, 2012). On the same note, it is essential to note that there might be different arrival routes in the location and visitors are also expected to arrive at different times where some 
would arrive prior to the event, and others would arrive on a later date when the event is at the climax, and these aspects just as well are considered. Following this knowledge, the temporal investments should be located at the entrance points for security services, and for food providing investments, they should be located where the population flow is high (Chen, Gong, Lawson, \& Bialostozky, 2010). The logistic challenges accrued to this temporal investment require expert investigation; this study also provides insight into the mechanisms that effectively resolve such challenges.

This study will provide and suggest a modeling procedure to create an operational plan to inform and guide temporal point investments for service delivery in large events in relevance to these complexities and challenges. The services of focus in this study are food and security provision, which must be competent in matching the available population. The model tabled targets to respond to the following questions: where are the specific resources targeted located? Where can the temporal services provision points be located? What is the expected population, and how it can be effectively served in relation to the number of the temporal service point and their capacity? Therefore, it is true that this research work aims to explain strategic system dynamics models that inform decision-making and management of the activities around the event (Khozium, 2012). Henceforth, the modeling results assume an algorithm and technique form for driving value in the event's constrained indicators.

\section{Literature Review}

This study work will exploit a 3-D consideration of a constrained event to determine the most effective placement and positioning of large-scale critical services temporal locations (Kwan, 2000). The number and capacity of the service points is also a critical point of interest and is carried out along the establishment's most effective time. As such, this research will focus on restrictions on space, time, and volume in consideration of the attendance population. It is also interesting to consider that the attending dignitaries will arrive in different transport modes. Therefore, a route used has such factors to consider apart from the mere population aspect. While some visitors will call in using busses, cars, or air, others would arrive by foot, and therefore the population served is not equivalent to the population flowing per unit time in a given route.

In Hajj and Olympic Games, among other large gatherings, requires a prior proficient optimal arrangement on resources serving points location for placement of the critical resources to ensure the maximum level of the opportunities provided by such population economic wise (Amro, 2012). Understanding the opportunities provided and the population demand along their routes and time of arrival is critical to informed decision-making on points of service delivery establishments that would facilitate optimal exploitation of these opportunities and the effective provision of the required resources such as food and security (Tayan, 2010).

Describing a temporal service point location problem can be addressed using five components. These components include the potential number of visitors who arrive or are expected and the route they are likely to follow as well as their mode of transport. The space available around the event area also counts on determining the serving capacity and the size of the establishment as well as the establishment points. Lastly, standard metrics further inform that distance and arrival time difference among the visitors also determines the investment's time and location (Desai, \& Ganatra, 2015).

This research has invested in the utilization of Geographic Information System (GIS) to identify and study existing relationships between the variables influencing optimal temporal investment location hence forms an essential part of this report on the study. Finally, this report highlights the limitations accrued to the literature review of this work as far as analysis of the prior mentioned variables is concerned because of their complexity and difficulty on integration.

\subsection{Research Questions}

What should be the optimal solution to determine the location of a service provision point in an event with large gatherings such that the number of visitors to the area or the facility is highly likely to fluctuate significantly over time? Unfortunately, this question remains unanswered.

\subsection{Research Aims}

The research at hand is dedicated to addressing the single prior highlighted research question. Generating answers to this research question depends on the research design and data collection methods and analysis in guidance of the aims and objectives aligned below.

- To introduce an emerging technique from findings on the analysis of previously available data on approaches to address issues around placement and location of temporal services delivery points for large gatherings of limited time with consideration of issues such as management of large gatherings within a limited time and area (Bonchi, Giannotti, Mazzanti, \& Pedreschi, 2003). 
- To confirm the hypothesis of whether the approach is universally applicable for other large gatherings.

Hopes are high that this report will be effective for application to events such as:

- Hajj in Saudi Arabia.

- Olympic Games.

- And other large gatherings.

\subsection{Theoretical Framework}

Advancements in planning and designing an operations venue linked to the Process Logistics Advanced Technical Optimization (PLATO), a project for the Athens 2004 Olympic games, resulted in a global renewed interest in the future application of such techniques in the future events. The proficiency in the management of this event, which has a large gathering in a limited space and a short period, made it ideal for application in other similar events with little modification to meet the event context's specifications (Douglas, Richardson, 2013).

Distinguished authors have invested their knowledge in writing and studies of optimization of services delivery to large gatherings for a short time in limited spatial areas. Theories developed dwells on linear programming mathematical concepts because of the effectiveness of this concept in handling such cases where facility placement is complicated due to the need to capture numerous characteristics on real-world locations. These characteristics include uncertainty of demand and providing a link between location models, layout, and network models.

Unfortunately, this literature review suffers blows on appropriate algorithms' limitations to address optimal placement and location of services point for temporal events with massive gatherings. The available models are not ideal for any event, and therefore assuming any of them requires informed engineering expertise (Martins, \& Gorschek, 2016). The required engineering expertise is used to solve theoretical complexities revolving around integers in linear programming on investments. However, this expertise and theories need to be added more variables involved in the research question problem. This results in a generation of a comprehensive solution integrating all the constraints, which are; limited time and space and population fluctuation, which influence services points' location.

\subsection{Important Notes}

- Hajj case study provides more capacity planning, design, and resource estimation data for services points' location, unlike the Olympic Games.

- Failure to solving facility placement problems by tabling and capturing characteristics in the real-world arena can be effectively handled by simulation and projections of quantified performance and accrued benefits (Heppenstall, Harland, Ross, \& Olner, 2013).

- It would be better to miss an explanation than variables in the simulation models (Hashim, 2014).

- There are limited documentation and explanations from existing models, hence developing models to explain hidden decision-making mechanisms.

- Understanding the dependencies and processes interconnections affecting information and organization require in-depth qualitative studies.

- The implementation process must be well understood in the stakeholders and management perspectives for a successful implementation procedure.

\section{Methodology}

This study focuses on Hajj case and assumes the following methodology:

\subsection{Spatial Location Analysis}

The study uses routes abstraction using GIS to predetermine locations of interest as far as transport and stop points are concerned, hence identifying pedestrian routes suitable for the locations.

Two-dimensional analysis (time and space) In this case, time and space are two major variables used in the abstraction of a model that helps to determine proceedings over a specific time frame and space. The process will involve projection or observation of pedestrians' volume flow during peak and off-peak hours. This will also facilitate the study of the population behavior on purchases and fluctuation.

\section{Spatial and population mobility analysis (time, space, and population)}

This modeling and abstraction allow the analysis of data associated with a dynamic population in a given space over a given time (Haghighati, \& Hassan, 2013). This 3-D simulation provides an understanding of acceptable and 
unacceptable crowding levels in a given location over a given period of time. The analysis also provides a projection on the pedestrian flow volume and other populations in different transit methods. It is essential to note that integrating spatial GIS facilitates simulation software development, which automates much of the visualized environment processes. Experts in the simulation field carried this out.

\subsection{Research Design}

- This research is a case study

- Involves 20 in-depth interviews where respondents include senior managers, young professionals, and review documented artifacts on Hajj analysis.

- Visual descriptions of the proposed location of the service point model using is created using appropriate software tools, with system dynamics and VensimPLE32 modelling software used to detailed map facilities and a simulation model. Therefore, use PASW SPSS modeller software to integrate different large dataset sources to have actual patterns and predictions. Synthetic data was generated randomly under certain circumstances. This then be loaded into the modeler.

- The primary findings are presented to inform of techniques and algorithms derived from constrained indicators.

\section{Research Results}

\subsection{Proposing the Simulation Algorithm}

Services delivery points locations are discrete and highly influenced by pedestrians' volume in the given line. The following key factors influence this:

Variable distance

GIS stores, manipulate, analyze, and present geographic data hence determining the optimal points of service delivery locations in the pedestrian path, which is the number of services points that should be established.

\subsubsection{Total Population Arrival}

The population in attendance can be retrieved from the GIS database. Services delivery points are established proportionally to the attendance number.

\subsubsection{Walk Time and Spatial Capacity}

System dynamics simulations will provide a better alternative to GIS in decision-making by guiding critical processes of clarification and evaluation of interactions between services point placement and population flow, hence developing proficient recommendations on procedures.

\subsubsection{Data Mining Algorithm Variables (Travel Time, Target Customers, Service Type, and Projected Population)}

Simulation on what service types are needed pose a significant advantage in predicting demand distribution from available estimates on incorporating several databases. This method seeks a solution by experimentation and combining various approaches targeting to deliver a profound solution that incorporates several approaches to the same problem.

\subsection{Results Analysis}

GIS examines three areas to determine and project the number of services points and their locations. These projections are the critical road map for the establishments where transport network, transport mode, population, and the stop-overs, are used to determine suitable locations. However, an aspect such as narrow road is the primary location disqualifying factor despite favor from the other variables. Time constraint, on the other hand, is used to determine the optimal time for the points location as well as the type of services that should be provided at different times. Following these results, population and time in the given space are the most significant determinants; hence walking speed and time, as well as peak and off-peak hours, are critical influencers of the point's location. It is essential to note that pedestrians' population can be estimated using numeric models such as density, walking flow, speed, and space traits.

\section{Finding}

The above results from the analysis of existing data gear in-depth insight into the research question, resulting in the development of three models essential in artifacts development by evaluating limited time and space and population dynamics fluctuation wise.

\section{When}

This question is answered better answered by findings from discrete-time event simulation for optimal periods, 
which utilizes the average population, at peak and off-peak, and the projected population on arrival data.

\section{Where}

GIS assumes the role of determining optimal locations for the service points along foot-paths hence their distribution as well.

\section{How many}

Each transport means is monitored on movement using dynamic system simulation (Chung, \& Shalaby, 2005). This provides essential information on the population flow per unit time in a given route and the number of pedestrians pass-by a given service point on their way to the event. Pedestrians interested in any service are likely to visit the pre-installed points, and their deficit would force for a queue. Waiting time and the queue's length provides important data in the projection of how many other points should be installed.

\section{The conceptual framework for the research problem}

The conceptual framework for placing service points under 3-D is explained as follows:

- $\quad$ Events - refer to temporal events over the duration of an event. It is looked at in the perspectives of time sequence, peak and off-peak time, and travel time is considered the primary output.

- $\quad$ Routes - refers to the spatial direction of location access. Distance and foot-paths are of interest in this case, while space capacity is a primary output (Teece, 2010).

- $\quad$ Arrivals - refers to attendance using various transport mechanisms. Include pedestrians, service points for the customers, and distribution demand is the most interesting output.

\section{The solution model}

The simulation model is the simplest possible for describing system behavior. It should utilize high-level visualization to support the solution on change of practices. Qualitative models for service points are more of practice and should be handled by an agent-based simulation technique to describe population distribution. Placements for service points must reach an acceptable number of people on flow and volume as predetermined by the geographical service point's placement findings from GIS analysis.

\section{Research contribution}

Placement location for a facility is a complicated encounter due to the numerous real-world features, which must be considered in solution finding. These features include network, locations, and layout models (Drira, Pierreval, \& Hajri-Gabouj, 2007). Interestingly, efficiency in solution provision to such aspects can be boosted by the use of modeling algorithms in research on pedestrians since it will ease the analysis of the collected data.

\section{Theoretical contribution}

- $\quad$ Developing an analytical technique to advise temporal service point positioning targeting to serve largescale gatherings in a limited space for a given period.

- $\quad$ To provide an analytical solution addressing limitations on time and space.

- $\quad$ To provide an analytical solution addressing critical services delivery to large gatherings.

\section{Empirical contribution:}

- $\quad$ Organizing large population events free of procedural crisis.

- Developing a practical event concept applicable in Saudi Arabia and other places and events with large gatherings other than Makkah.

\section{Discussion}

Success in service provision in big gatherings depends on the management's proficiency to use simulation and informed decision-making on location and number of services points to establish (Ginter, Duncan, \& Swayne, 2018). GIS is one of these prominent tools and should be used to analyze and inform an optimal number of locations to establish where to establish them in terms of spacing and each service provision points' capacity depending on the gatherings' size.

The use of GIS and simulation by systems provides concrete answers on when the service points should be established with proficiency on timing accounting on the fact visitors arrive in the event scene at different times. At the same time, some visitors arrive significantly earlier before the event, others arrive immediately on the event's kick-off, and others arrive when the event is at the peak (Kersulić, Perić, \& Wise, 2020). These modelings also provide essential information to answer how many service points are optimal to the size of the gathering 
expected. This decision is further informed on the location of the service points accounting on the route and transport modes used by the gathering where the pedestrian population is the critical aspect of consideration, and most of the service points should be established where the pedestrian population is the highest and must have a slow speed of movement (Greenwell, Danzey-Bussell, \& Shonk, 2019). The simulation and modeling processes for service point establishments must be based on 3-D aspects of space limitations, limitations in stay time, and the gathering size.

\section{Conclusion}

Hajj and Olympic games are among the global events that table temporal opportunities to investors. These opportunities include the provision of essential services, such as food and security. However, the exploitation of these opportunities to the maximum relies on prowess in determining the capacity and the optimum number of the service points that should be established. Size of the crowd is important in this analysis, but the size of the pedestrian population is even more important. Establishing service points require proficiency to reap the optimum benefit. This proficiency is the application of time, crowd size in a limited area, and the crowd's walk time in simulation application to determine the critical areas where given service points should be established. Otherwise, the poor establishment of service points will result in a logistics crisis. In conclusion, the management and planners of any big event in the sense of the gathering's size are obliged to make arrangements on the proficient establishment of service points. This proficiency is drawn from the use of simulation and modeling using computing software to ensure sound planning on where these service points, their service capacity, and their number as well as their separation distance.

\section{Recommendation}

Large gatherings like that in the Olympic Games and Hajj in Makkah require uninterrupted services provision such as food, water, and security. The service provision points targeting these events are temporal, and therefore investment in them should also be temporal, but with optimum exploitation of the opportunities availed. The gathering size should be a primary determinant of the nature, size, capacity, and the number of the services points to be established. Proficiency in this decision is based on prowess to use modeling and simulation with the help of GIS applications.

\section{Limitations}

This analytical work on temporal business exploitation to the optimum level as far as large crowd is concerned and has a limited stay time suffers several blows. Making an informed decision on this scenario requires the availability of a massive volume of data on the aspect which is not in existence. Also, simulation knowledge and resources are also limited. Another limitation on the development of this analytical work is the variability of consumption behavior of the large gathering at hand since they have different backgrounds, tastes, and preferences.

\section{Acknowledgements}

First, I thank Almighty and Merciful Allah who helped me to complete this article. I would also like to express my sincere gratitude to all authors of journals who have provided the constructive critique to refine the research direction. The completion of this research work would not be possible without their valuable knowledge and guidelines.

Additionally, I would like to acknowledge to University of Manchester and King Abdel Aziz University for providing excellent research facilities and financial support. I would like to thank ex-supervisors for their constant encouragement, and supportive guidance. Thanks to all.

\section{References}

Almunawar, M. N., Susanto, H., \& Anshari, M. (2013). A cultural transferability on IT business application: iReservation system. Journal of Hospitality and Tourism Technology, 4(2), 155-176. https://doi.org/10.1108/JHTT-05-2012-0015

Amro, A. (2012). Pilgrims “Hajj”. Tracking System (e-Mutawwif), 5(9), 437-446.

Beis, D. A., Loucopoulos, P., Pyrgiotis, Y., \& Zografos, K. G. (2006). PLATO helps Athens win gold: Olympic game knowledge modeling for organizational change and resource management. Interfaces, 36(1), 26-42. https://doi.org/10.1287/inte.1060.0189

Bonchi, F., Giannotti, F., Mazzanti, A., \& Pedreschi, D. (2003). Exante: Anticipated data reduction in constrained pattern mining. In Knowledge Discovery in Databases: PKDD 2003 (pp. 59-70). Springer Berlin Heidelberg. 
Brdesee, H. S. (2013). Exploring Factors Impacting E-Commerce Adoption in Tourism Industry in Saudi Arabia.

Chen, C., Gong, H., Lawson, C., \& Bialostozky, E. (2010). Evaluating the feasibility of a passive travel survey collection in a complex urban environment: Lessons learned from the New York City case study. Transportation Research Part A, 44, 830-840. https://doi.org/10.1016/j.tra.2010.08.004

Chung, E., \& Shalaby, A. (2005). A trip reconstruction tool for GPS-based personal travel surveys. Transportation Planning and Technology, 28(5), 381-401. https://doi.org/10.1080/03081060500322599

Desai, N. A., \& Ganatra, A. (2015). Buying Scenario and Recommendation of Purchase by Constraint Based Sequential Pattern Mining from Time Stamp Based Sequential Dataset. Procedia Computer Science, 45, 166175. https://doi.org/10.1016/j.procs.2015.03.113

Douglas, B. R. (2013). Real-Time Space-Time Integration in GIScience and Geography. Annals of the Association of American Geographers, 103(5), 1062-1071. https://doi.org/10.1080/00045608.2013.792172

Drira, A., Pierreval, H., \& Hajri-Gabouj, S. (2007). Facility layout problems: A survey. Annual Reviews in Control, 31(2), 255-267. https://doi.org/10.1016/j.arcontrol.2007.04.001

Ginter, P. M., Duncan, W. J., \& Swayne, L. E. (2018). The strategic management of health care organizations. John Wiley \& Sons.

Greenwell, T. C., Danzey-Bussell, L. A., \& Shonk, D. J. (2019). Managing sport events. Human Kinetics.

Haghighati, R., \& Hassan, A. (2013). Modeling The Flow of Crowd During Tawaf At Masjid Al-Haram. Journal Mekanikal, (36), 2-18.

Hashim, H. A. (2014). A Study of Adoption and Acceptance of e-Umrah System in Saudi Arabia: Overcoming Infrastructure Barriers and Limitations.

Heppenstall, A. J., Harland, K., Ross, A. N., \& Olner, D. (2013). Simulating Spatial Dynamics and Processes in a Retail Gasoline Market: An Agent-Based Modeling Approach. Transactions in GIS, 17(5), 661-682. https://doi.org/10.1111/tgis.12027

Kersulić, A., Perić, M., \& Wise, N. (2020). Assessing and Considering the Wider Impacts of Sport-Tourism Events: A Research Agenda Review of Sustainability and Strategic Planning Elements. Sustainability, 12(11), 4473. https://doi.org/10.3390/su12114473

Khozium, M. O. (2012). A Hybrid Intelligent Information System for the Administration of Massive Mass of Hajjis.

Kwan, M. P. (2000). Interactive geovisualization of activity-travel patterns using three-dimensional geographical information systems: a methodological exploration with a large data set. Transportation Research Part C: Emerging Technologies, 8(1), 185-203. https://doi.org/10.1016/S0968-090X(00)00017-6

Loucopoulos, P., Zografos, K., \& Prekas, N. (2003). Requirements elicitation for the design of venue operations for the Athens 2004 Olympic Games. In Requirements Engineering Conference, 2003. Proceedings. 11th IEEE International (pp. 223-232). IEEE. https://doi.org/10.1109/ICRE.2003.1232753

Marketing, A. P., \& Arabia, S. (2012). Consumer Behavior and Retail Market Consumerism in KSA. Consumer Behavior and Retail Market Consumerism in KSA, 3(11), 1-14.

Martins, L. E. G., \& Gorschek, T. (2016). Requirements engineering for safety-critical systems: A systematic literature review. Information and Software Technology, 75, 71-89. https://doi.org/10.1016/j.infsof.2016.04.002

Tayan, O. (2010). A Proposed Model for Optimizing the Flow of Pilgrims between Holy Sites During Hajj Using Traffic Congestion Control.

Teece, D. J. (2010). Business models, business strategy and innovation. Long Range Planning, 43(2-3), 172-194. https://doi.org/10.1016/j.lrp.2009.07.003

\section{Copyrights}

Copyright for this article is retained by the author(s), with first publication rights granted to the journal.

This is an open-access article distributed under the terms and conditions of the Creative Commons Attribution license (http://creativecommons.org/licenses/by/4.0/). 